\title{
Analysis of a pressure-stabilized finite element approximation of the stationary Navier-Stokes equations
}

\author{
Ramon Codina $^{1}$, Jordi Blasco ${ }^{2}$ \\ 1 Universitat Politècnica de Catalunya, Jordi Girona 1-3, Edifici C1, 08034 Barcelona, \\ Spain; e-mail: ramon.codina@upc.es \\ 2 e-mail: blasco@ma1.upc.es
}

Received May 25, 1998 / Revised version received August 31, 1999 /

Published online July 12, 2000 - (C) Springer-Verlag 2000

Summary. The purpose of this paper is to analyze a finite element approximation of the stationary Navier-Stokes equations that allows the use of equal velocity-pressure interpolation. The idea is to introduce as unknown of the discrete problem the projection of the pressure gradient (multiplied by suitable algorithmic parameters) onto the space of continuous vector fields. The difference between these two vectors (pressure gradient and projection) is introduced in the continuity equation. The resulting formulation is shown to be stable and optimally convergent, both in a norm associated to the problem and in the $L^{2}$ norm for both velocities and pressure. This is proved first for the Stokes problem, and then it is extended to the nonlinear case. All the analysis relies on an inf-sup condition that is much weaker than for the standard Galerkin approximation, in spite of the fact that the present method is only a minor modification of this.

Mathematics Subject Classification (1991): 65N30, 76D05

\section{Introduction}

One of the most important subjects of research in the finite element approximation of the incompressible Navier-Stokes equations concerns the velocity-pressure interpolation. When the standard Galerkin formulation is used, the spaces chosen for the approximation of these variables have to satisfy the classical inf-sup or Babuška-Brezzi condition (see, e.g., [6]). Much 


\section{Statement of the problem}

\subsection{The stationary incompressible Navier-Stokes equations}

Let us consider the classical stationary Navier-Stokes equations for an incompressible fluid. Let $\Omega$ be an open, bounded and polyhedral domain of $\mathbb{R}^{d}$, where $d=2$ or 3 is the number of space dimensions, and $\Gamma=\partial \Omega$ its boundary. The Navier-Stokes problem consists in finding a velocity $\boldsymbol{u}$ and a scaled pressure $p$ (the kinematic pressure divided by the kinematic viscosity) such that

$$
\begin{aligned}
\lambda\left[\frac{1}{2}(\nabla \cdot \boldsymbol{u}) \boldsymbol{u}+(\boldsymbol{u} \cdot \nabla) \boldsymbol{u}\right]-\Delta \boldsymbol{u}+\nabla p & =\lambda \boldsymbol{f} \quad \text { in } \Omega, \\
\nabla \cdot \boldsymbol{u} & =0 \quad \text { in } \Omega, \\
\boldsymbol{u} & =\mathbf{0} \quad \text { on } \Gamma
\end{aligned}
$$

where $\lambda$ is the inverse of the kinematic viscosity and $f$ is the force vector. We have considered the homogeneous Dirichlet boundary condition (3) for simplicity. The expression adopted for the nonlinear term will simplify the analysis, although for the continuous problem it could be replaced by $(\boldsymbol{u} \cdot \nabla) \boldsymbol{u}$.

To write the weak form of problem (1)-(3) we need to introduce some notation. As usual, we denote by $H^{m}(\omega)$ the Sobolev space of $m$-th order in a set $\omega$, consisting of functions whose distributional derivatives of order up to $m$ belong to $L^{2}(\omega)$, and by $H_{0}^{1}(\omega)$ the subspace of $H^{1}(\omega)$ of functions with zero trace on $\Gamma$. A bold character is used for the vector counterpart of these spaces. The $L^{2}$ scalar product is denoted by $(, \cdot) \omega$, and the $H^{m}$ norm by $\|\cdot\|_{m, \omega}$. The subscript $m$ is omitted when $m=0$ and so is $w$ when it is $\Omega$.

\section{Let us now consider the spaces}

gister for free at https//www.scipedia.com to download the version withou

$$
V=H_{0}^{1}(\Omega),
$$$$
Q=\left\{q \in L^{2}(\Omega) \mid \int_{\Omega} q \mathrm{~d} \Omega=0\right\} \text {, }
$$

and the forms

$$
\begin{aligned}
a(\boldsymbol{u}, \boldsymbol{v}) & :=(\nabla \boldsymbol{u}, \nabla \boldsymbol{v}), \\
b(q, \boldsymbol{v}) & :=(q, \nabla \cdot \boldsymbol{v}), \\
c(\boldsymbol{u}, \boldsymbol{v}, \boldsymbol{w}) & :=\left(\frac{1}{2}(\nabla \cdot \boldsymbol{u}) \boldsymbol{v}+(\boldsymbol{u} \cdot \nabla) \boldsymbol{v}, \boldsymbol{w}\right),
\end{aligned}
$$

with $\boldsymbol{u}, \boldsymbol{v}, \boldsymbol{w} \in \boldsymbol{V}$ and $q \in Q$. All these forms are continuous and $c$ is skew-symmetric in its last two arguments, that is

$$
c(\boldsymbol{u}, \boldsymbol{v}, \boldsymbol{w})=-c(\boldsymbol{u}, \boldsymbol{w}, \boldsymbol{v}) .
$$


Definition 1 (a) The stationary incompressible Navier-Stokes equations (1)-(3) are called regular if $[\boldsymbol{u}, p] \in \boldsymbol{V} \cap \boldsymbol{H}^{2}(\Omega) \times Q \cap H^{1}(\Omega)$ whenever $\boldsymbol{f} \in \boldsymbol{L}^{2}(\Omega)$, and there exists a constant $C_{r}>0$ such that

$$
\|\boldsymbol{u}\|_{2}+\|p\|_{1} \leq C_{r}\|\boldsymbol{f}\| .
$$

(b) Let $k \geq 1$ be an integer. Solutions $[\boldsymbol{u}, p]$ to (13) are called $k$-regular if $[\boldsymbol{u}, p] \in \boldsymbol{V} \cap \boldsymbol{H}^{k+1}(\Omega) \times Q \cap H^{k}(\Omega)$.

It is known for example that problem (1)-(3) is regular when $\Omega$ is a bounded and convex polygon when $d=2$ or when $\Omega$ is of class $\mathcal{C}^{2}$ in any space dimension [20]. Note that 1-regular solutions are not necessarily regular since the shift (16) may not hold.

\subsection{Finite element discretization}

Let $\mathcal{T}_{h}$ denote a finite element partition of the domain $\Omega$ of diameter $h$. For simplicity, we assume that all the element domains $K \in \mathcal{T}_{h}$ are the image of a reference element $\hat{K}$ through a polynomial mapping $\boldsymbol{F}_{K}$, affine for simplicial elements, bilinear for quadrilaterals and trilinear for hexahedra. On $\hat{K}$ we define the polynomial spaces $R_{k}(\hat{K})$ where, as usual, $R_{k}=P_{k}$ for simplicial elements and $R_{k}=Q_{k}$ for quadrilaterals and hexahedra. The finite element spaces we need are 17
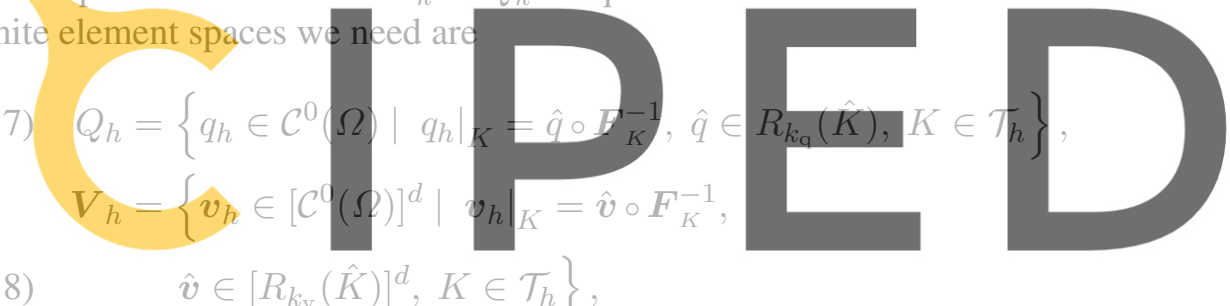

(18)

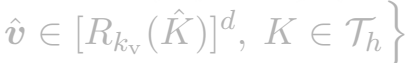

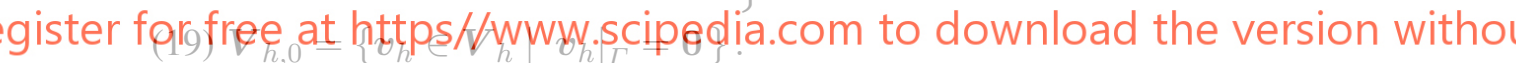

Notice that all these finite element spaces are referred to the same partition and are made up with continuous functions.

The standard Galerkin finite element counterpart of problem (13) can now be written as follows: find $\left[\boldsymbol{u}_{h}, p_{h}\right] \in \boldsymbol{V}_{h, 0} \times Q_{h}$ such that (20) $\lambda c\left(\boldsymbol{u}_{h}, \boldsymbol{u}_{h}, \boldsymbol{v}_{h}\right)+a\left(\boldsymbol{u}_{h}, \boldsymbol{v}_{h}\right)-b\left(p_{h}, \boldsymbol{v}_{h}\right)+b\left(q_{h}, \boldsymbol{u}_{h}\right)=\lambda\left\langle\boldsymbol{f}, \boldsymbol{v}_{h}\right\rangle$,

for all $\left[\boldsymbol{v}_{h}, q_{h}\right] \in \boldsymbol{V}_{h, 0} \times Q_{h}$. It is well known that if this formulation is used the velocity and pressure finite element spaces must satisfy the discrete counterpart of the inf-sup condition (12). For the finite element spaces (17)(19) this happens if $k_{\mathrm{v}}=k_{\mathrm{q}}+1$, i.e., for Taylor-Hood type elements [2,5, 18] This condition is not necessary using the method described next. 
Let $\alpha_{K}, K \in \mathcal{T}_{h}$, be a family of mesh parameters depending on the element sizes $h_{K}$. The modification of problem (20) that we propose is: find $\left[\boldsymbol{u}_{h}, p_{h}, \tilde{\boldsymbol{u}}_{h}\right] \in \boldsymbol{V}_{h, 0} \times Q_{h} \times \boldsymbol{V}_{h}$ such that

$$
\begin{aligned}
\lambda c\left(\boldsymbol{u}_{h}, \boldsymbol{u}_{h}, \boldsymbol{v}_{h}\right)+a\left(\boldsymbol{u}_{h}, \boldsymbol{v}_{h}\right)-b\left(p_{h}, \boldsymbol{v}_{h}\right) & =\lambda\left\langle\boldsymbol{f}, \boldsymbol{v}_{h}\right\rangle, \\
\sum_{K} \alpha_{K}^{2}\left(\nabla p_{h}, \nabla q_{h}\right)_{K}-\sum_{K} \alpha_{K}\left(\tilde{\boldsymbol{u}}_{h}, \nabla q_{h}\right)_{K}+b\left(q_{h}, \boldsymbol{u}_{h}\right) & =0 \\
-\sum_{K} \alpha_{K}\left(\nabla p_{h}, \tilde{\boldsymbol{v}}_{h}\right)_{K}+\left(\tilde{\boldsymbol{u}}_{h}, \tilde{\boldsymbol{v}}_{h}\right) & =0
\end{aligned}
$$

for all $\left[\boldsymbol{v}_{h}, q_{h}, \tilde{\boldsymbol{v}}_{h}\right] \in \boldsymbol{V}_{h, 0} \times Q_{h} \times \boldsymbol{V}_{h}$.

For a function $q \in H^{1}(\Omega)$ let us define $\nabla^{h} q$ by

$$
\left.\nabla^{h} q\right|_{K}=\left.\alpha_{K} \nabla q\right|_{K}, \quad K \in \mathcal{T}_{h}
$$

From (23) it is seen that $\tilde{u}_{h}$ is the projection of $\nabla^{h} p_{h}$ onto $V_{h}$.

Let us also introduce the form

$$
\begin{aligned}
\mathcal{B}( & \left.\lambda ; \boldsymbol{u}_{h}, p_{h}, \tilde{\boldsymbol{u}}_{h} ; \boldsymbol{v}_{h}, q_{h}, \tilde{\boldsymbol{v}}_{h}\right) \\
:= & \lambda c\left(\boldsymbol{u}_{h}, \boldsymbol{u}_{h}, \boldsymbol{v}_{h}\right)+a\left(\boldsymbol{u}_{h}, \boldsymbol{v}_{h}\right)-b\left(p_{h}, \boldsymbol{v}_{h}\right)+b\left(q_{h}, \boldsymbol{u}_{h}\right) \\
& +\left(\nabla^{h} p_{h}-\tilde{\boldsymbol{u}}_{h}, \nabla^{h} q_{h}-\tilde{\boldsymbol{v}}_{h}\right) .
\end{aligned}
$$

(25)

Problem (21)-(23) can be written now as: find $\left[\boldsymbol{u}_{h}, p_{h}, \tilde{\boldsymbol{u}}_{h}\right] \in \boldsymbol{V}_{h, 0} \times$

\section{$Q_{h} \times V_{h}$ such that}

(26)

for all $\left[v_{h}, q_{h}, \tilde{v}_{h}\right] \in V h, 0$
Let us describe now th

element partitions $\left\{\mathcal{T}_{h}\right\}_{h>0}$. First of all, we assume that it is non-degenerate,

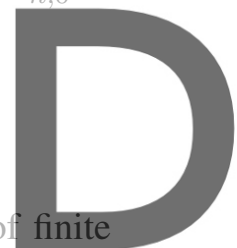

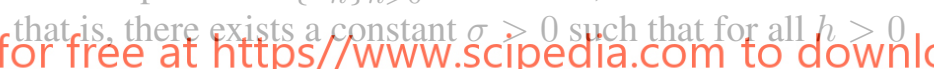

$$
\operatorname{diam}\left(B_{K}\right) \geq \sigma \operatorname{diam}(K) \forall K \in \mathcal{T}_{h},
$$

where $B_{K}$ is the largest ball contained in $K \in \mathcal{T}_{h}$. Condition (27) is needed in order to have the following inverse estimates (see, e.g., [3]): there exist positive constants $C_{1}$ and $C_{2}$ such that

$$
\begin{aligned}
\left\|v_{h}\right\|_{1, K} & \leq C_{1} h_{K}^{-1}\left\|v_{h}\right\|_{0, K}, \\
\left\|v_{h}\right\|_{L^{\infty}(K)} & \leq C_{2} h_{K}^{-d / 2}\left\|v_{h}\right\|_{0, K},
\end{aligned}
$$

where $v_{h}$ is a function of any of the finite element spaces (17)-(19).

From now onwards we use $C$, possibly with subscripts, to denote a positive constant independent of the mesh size and $\lambda$, not necessarily the same at different occurrences. 
Since we always have that

$$
\left\|v_{h}\right\|_{0, K} \leq C h_{K}^{d / 2}\left\|v_{h}\right\|_{L^{\infty}(K)}
$$

from (29) it follows that

$$
C_{1} h_{K}^{d / 2}\left\|v_{h}\right\|_{L^{\infty}(K)} \leq\left\|v_{h}\right\|_{0, K} \leq C_{2} h_{K}^{d / 2}\left\|v_{h}\right\|_{L^{\infty}(K)}
$$

Let $n_{\text {nod }}$ be the number of nodes per element and $N_{i}$ the shape function associated to the $i$-th node. If $v_{i}$ is the value of a function $v_{h}$ at this node and we are given a set of nodal parameters $\beta=\left\{\beta_{1}, \ldots, \beta_{n_{\text {nod }}}\right\}$, we define

$$
\Pi_{K}\left(\beta v_{h}\right):=\sum_{i=1}^{n_{\text {nod }}} N_{i} \beta_{i} v_{i}
$$

Let $\max _{K}|\beta|$ be the maximum of the absolute value of the nodal parameters $\beta$ in an element $K$. It is easy to see that

$$
\left\|\Pi_{K}\left(\beta v_{h}\right)\right\|_{L^{\infty}(K)} \leq C \max _{K}|\beta|\left\|v_{h}\right\|_{L^{\infty}(K)}
$$

which, by virtue of (30), implies that

$(32)$

We shall need a rather technical condition on th
$\alpha_{K}$, which is reflected by a condition on $\left\{\mathcal{T}_{h}\right.$

on $h_{K}$, as we shall see. Let $M_{i}$ be a macroelement

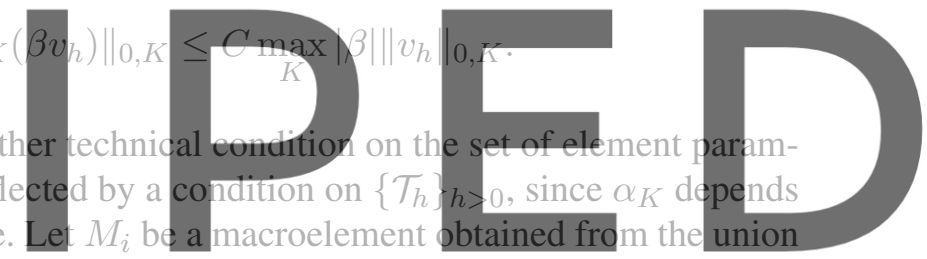

of the elements to which a node $i$ belongs. Let us define

gister for free at https//www.scipedia.com to download the version withou (33)

$$
\bar{\alpha}_{i}:=\frac{1}{\operatorname{meas} M_{i}} \sum_{K \subset M_{i}} \operatorname{meas} K \alpha_{K}
$$

which is nothing but a weighted average of the parameters $\alpha_{K}$. We need this average to converge to these parameters as the mesh is refined. More precisely, we need a continuously graded family of meshes, a concept introduced in the following:

Definition 2 The family of finite element meshes $\left\{\mathcal{T}_{h}\right\}_{h>0}$ is continuously graded if there exists a function $\delta=\delta(h)$, tending to 0 as $h \rightarrow 0$, such that

$$
\max _{K \in \mathcal{T}_{h}}\left(\max _{K}\left|1-\frac{\bar{\alpha}}{\alpha_{K}}\right|\right) \leq \delta(h) .
$$


This condition is less restrictive than the quasi-uniformity of the family $\left\{\mathcal{T}_{h}\right\}_{h>0}$. It allows for instance local mesh refinement. What it does not permit, for example, is a constant ratio between the sizes of the elements sharing a fixed nodal point as the mesh is refined. From the practical point of view this is not a restriction, since the element sizes given to mesh generators are usually continuous functions obtained from the interpolation of element sizes at certain given points of the computational domain.

Condition (34) implies in particular that

$$
\max _{K}|\bar{\alpha}| \leq(1+\delta(h)) \alpha_{K}
$$

Our stability and convergence analysis of the following sections will be strongly based on a decomposition of the pressure gradient that we describe next. Let $\nabla^{h} Q_{h}$ denote the space of vector functions in $\boldsymbol{L}^{2}(\Omega)$ which are of the form $\nabla^{h} q_{h}$, with $q_{h} \in Q_{h}$, and consider the vector space

$$
E_{h}:=V_{h}+\nabla^{h} Q_{h}=E_{h, 1} \oplus E_{h, 2} \oplus E_{h, 3},
$$

where $E_{h, i}, i=1,2,3$, are three mutually $L^{2}$ orthogonal subspaces defined as

(37) $\boldsymbol{E}_{h, 1}:=\boldsymbol{V}_{h, 0}$,

(38)

(39)

Let us denote by $P$

$P_{h, i}+P_{h, j}, i, j=1,2,3$

to prove that the pressure gradient in problem (26) independently the three terms in the decomposition

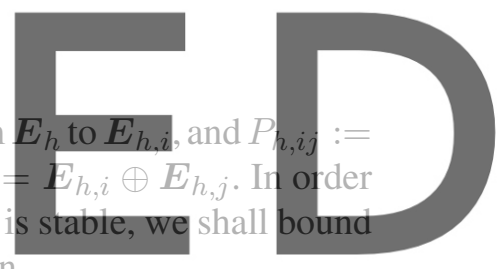

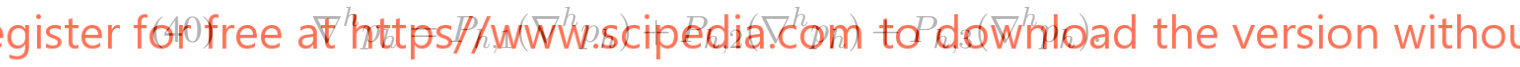

To obtain error estimates for solutions of problem (26) we shall make use of the approximation properties of the spaces $\overline{\boldsymbol{V}}_{h, 0}, Q_{h}$ and $\overline{\boldsymbol{V}}_{h}$. These can be written as follows. If $\boldsymbol{v} \in \boldsymbol{H}^{r}(\Omega) \cap \boldsymbol{V}, r \geq 1$, and $q \in H^{s}(\Omega) \cap Q$, $s \geq 1$, there exist $I_{h, 1}(\boldsymbol{v}) \in \boldsymbol{V}_{h, 0}, I_{h, 2}(q) \in Q_{h}$ and $I_{h, 3}(\nabla q) \in \boldsymbol{V}_{h}$ such that

$$
\begin{gathered}
\left\|\boldsymbol{v}-I_{h, 1}(\boldsymbol{v})\right\|_{m} \leq C_{1} h^{k_{1}}\|\boldsymbol{v}\|_{k_{1}}, k_{1}=\min \left\{r, k_{\mathrm{v}}+1\right\}-m, \\
\left\|q-I_{h, 2}(q)\right\|_{m} \leq C_{2} h^{k_{2}}\|q\|_{k_{2}}, k_{2}=\min \left\{s, k_{\mathrm{q}}+1\right\}-m, \\
\left\|\nabla^{h} q-I_{h, 3}\left(\nabla^{h} q\right)\right\|_{m, K} \leq C_{3} h^{k_{3}}\left\|\nabla^{h} q\right\|_{k_{3}, K} \\
k_{3}=\min \left\{s-1, k_{\mathrm{v}}+1\right\}-m
\end{gathered}
$$

Notice that the last estimate is local, since $\nabla^{h} q$ is in general discontinuous. 
for a constant $C$ independent of $h$.

Proof. Since the problem is linear and finite-dimensional, it is enough to prove that (47) holds. From the definition of the bilinear form $\mathcal{B}$ for $\lambda=0$ it is easy to see that

$$
\begin{aligned}
\mathcal{B}\left(0 ; \boldsymbol{u}_{h}, p_{h}, \tilde{\boldsymbol{u}}_{h} ; \boldsymbol{u}_{h}, p_{h}, \tilde{\boldsymbol{u}}_{h}\right) & =a\left(\boldsymbol{u}_{h}, \boldsymbol{u}_{h}\right)+\left\|\nabla^{h} p_{h}-\tilde{\boldsymbol{u}}_{h}\right\|^{2} \\
& \leq \lambda\|\boldsymbol{f}\|_{-1}\left\|\boldsymbol{u}_{h}\right\|_{1} .
\end{aligned}
$$

From the coercivity of the bilinear form $a$ (see (11)) it follows that

$$
\left\|\boldsymbol{u}_{h}\right\|_{1} \leq \lambda K_{a}^{-1}\|\boldsymbol{f}\|_{-1} .
$$

On the other hand, (23) can now be written as $\tilde{\boldsymbol{u}}_{h}=P_{h, 12}\left(\nabla^{h} p_{h}\right)$, and therefore from (48) it follows that

$$
\left\|P_{h, 3}\left(\nabla^{h} p_{h}\right)\right\|^{2}=\left\|\nabla^{h} p_{h}-\tilde{\boldsymbol{u}}_{h}\right\|^{2} \leq \lambda\|\boldsymbol{f}\|_{-1}\left\|\boldsymbol{u}_{h}\right\|_{1},
$$

and, from estimate (49),

$$
\left\|P_{h, 3}\left(\nabla^{h} p_{h}\right)\right\| \leq K_{a}^{-1 / 2} \lambda\|\boldsymbol{f}\|_{-1} .
$$

If $\boldsymbol{v}_{h} \in \boldsymbol{E}_{h}$, let us define $\Pi\left(\bar{\alpha} \boldsymbol{v}_{h}\right)$ by $\left.\Pi\left(\bar{\alpha} \boldsymbol{v}_{h}\right)\right|_{K}=\Pi_{K}\left(\bar{\alpha} \boldsymbol{v}_{h}\right)$, with $\Pi_{K}$ defined in (31). We have that

$$
\begin{aligned}
\left\|P_{h, 1}\left(\nabla^{h} p_{h}\right)\right\|^{2} & =\sum_{K}\left(\alpha_{K} \nabla p_{h}, P_{h, 1}\left(\nabla^{h} p_{h}\right)\right)_{K} \\
& =\left(\nabla p_{h}, \Pi\left(\bar{\alpha} P_{h, 1}\left(\nabla^{h} p_{h}\right)\right)\right) \\
& +\sum_{K}\left(\nabla p_{h}, \alpha_{K} P_{h, 1}\left(\nabla^{h} p_{h}\right)-\Pi_{K}\left(\bar{\alpha} P_{h, 1}\left(\nabla^{h} p_{h}\right)\right)\right)_{K} \\
& =: T_{1}+T_{2} .
\end{aligned}
$$

Let us bound the terms $T_{1}$ and $T_{2}$. From the momentum equation (21) (without the nonlinear term) we have that

$$
\begin{aligned}
T_{1} & =\lambda\left\langle\boldsymbol{f}, \Pi\left(\bar{\alpha} P_{h, 1}\left(\nabla^{h} p_{h}\right)\right)\right\rangle-a\left(\boldsymbol{u}_{h}, \Pi\left(\bar{\alpha} P_{h, 1}\left(\nabla^{h} p_{h}\right)\right)\right) \\
& \leq\left[\lambda\|\boldsymbol{f}\|_{-1}+N_{a}\left\|\boldsymbol{u}_{h}\right\|_{1}\right]\left\|\Pi\left(\bar{\alpha} P_{h, 1}\left(\nabla^{h} p_{h}\right)\right)\right\|_{1} .
\end{aligned}
$$

Using the inverse estimate (28) and inequalities (32) and (35), together with assumption (46) on $\alpha_{K}$, we obtain

$$
\left\|\Pi\left(\bar{\alpha} P_{h, 1}\left(\nabla^{h} p_{h}\right)\right)\right\|_{1}^{2}=\sum_{K}\left\|\Pi_{K}\left(\bar{\alpha} P_{h, 1}\left(\nabla^{h} p_{h}\right)\right)\right\|_{1, K}^{2}
$$




$$
\begin{aligned}
& \leq \sum_{K} \frac{C}{h_{K}^{2}}\left\|\Pi_{K}\left(\bar{\alpha} P_{h, 1}\left(\nabla^{h} p_{h}\right)\right)\right\|_{0, K}^{2} \\
& \leq \sum_{K} \frac{C}{h_{K}^{2}} \max _{K}|\bar{\alpha}|^{2}\left\|P_{h, 1}\left(\nabla^{h} p_{h}\right)\right\|_{0, K}^{2} \\
& \leq C\left\|P_{h, 1}\left(\nabla^{h} p_{h}\right)\right\|^{2} .
\end{aligned}
$$

Using this in (52), together with inequalities (32) and (49), it follows that

$$
T_{1} \leq C \lambda\|\boldsymbol{f}\|_{-1}\left\|P_{h, 1}\left(\nabla^{h} p_{h}\right)\right\| .
$$

To bound $T_{2}$ observe first that

$$
\begin{aligned}
& \frac{1}{\alpha_{K}}\left(\alpha_{K} P_{h, 1}\left(\nabla^{h} p_{h}\right)-\Pi_{K}\left(\bar{\alpha} P_{h, 1}\left(\nabla^{h} p_{h}\right)\right)\right) \\
& \quad=\Pi_{K}\left(\beta^{K} P_{h, 1}\left(\nabla^{h} p_{h}\right)\right)
\end{aligned}
$$

with $\beta_{i}^{K}=1-\bar{\alpha}_{i} / \alpha_{K}$, and therefore we obtain, using inequality (32), assumption (34), the weak inf-sup condition (44) and the bound (50),

$$
\begin{aligned}
T_{2} & =\sum_{K}\left(\alpha_{K} \nabla p_{h}, \Pi_{K}\left(\beta^{K} P_{h, 1}\left(\nabla^{h} p_{h}\right)\right)\right)_{K} \\
& \leq\left\|\nabla^{h} p_{h}\right\|\left(\sum_{K}\left\|\Pi_{K}\left(\beta^{K} P_{h, 1}\left(\nabla^{h} p_{h}\right)\right)\right\|_{0, K}^{2}\right)^{1 / 2} \\
& \leq C \delta(h)\left\|\nabla^{h} p_{h}\right\|\left\|P_{h, 1}\left(\nabla^{h} p_{h}\right)\right\| \\
& \leq C \delta(h)\left(\left\|P_{h, 1}\left(\nabla^{h} p_{h}\right)\right\|^{2}+\left\|P_{h, 1}\left(\nabla^{h} p_{h}\right)\right\|\left\|P_{h, 3}\left(\nabla^{h} p_{h}\right)\right\|\right) \\
& \leq C \delta(h)\left\|P_{h, 1}\left(\nabla^{h} p_{h}\right)\right\|^{2}+C \lambda\|\boldsymbol{f}\|_{-1}\left\|P_{h, 1}\left(\nabla^{h} p_{h}\right)\right\| .
\end{aligned}
$$

Using inequalities (54) and (56) in (51) we have that

$$
(1-C \delta(h))\left\|P_{h, 1}\left(\nabla^{h} p_{h}\right)\right\|^{2} \leq C \lambda\|\boldsymbol{f}\|_{-1}\left\|P_{h, 1}\left(\nabla^{h} p_{h}\right)\right\|,
$$

which for $h$ small enough implies that

$$
\left\|P_{h, 1}\left(\nabla^{h} p_{h}\right)\right\| \leq C \lambda\|\boldsymbol{f}\|_{-1} .
$$

This, together with (50) and the weak stability condition (44) implies the bound for $\nabla^{h} p_{h}$ in (47). Finally, the bound for $\tilde{\boldsymbol{u}}_{h}$ follows from the fact that $\tilde{\boldsymbol{u}}_{h}=P_{h, 12}\left(\nabla^{h} p_{h}\right)$. 
A similar stability estimate to that of (47) can be obtained replacing $\alpha_{K}$ by $h_{K}$ and using only the condition $\alpha_{K} \geq \alpha_{0} h_{K}$ instead of (46) (see [10]). Observe that we have only used the fact that $\alpha_{K} \leq \alpha_{1} h_{K}$ in (53). However, this part of assumption (46) is needed also for the error estimate presented next, which establishes convergence of the solution of problem (26) to the solution of the continuous Stokes problem in the norm in which stability has been proven, that is, in the $H^{1}$ norm for the velocity and the $L^{2}$ norm of the mesh dependent pressure gradient defined in (24) and its projection onto $\boldsymbol{V}_{h}$. This is what can be called the 'natural' norm of the method.

Theorem 2 Under the same conditions as in Theorem 1, suppose also that the Stokes problem is $k$-regular, with $k \geq 1$. Then, for $h$ small enough, the solution of problem (26) satisfies the error estimate

$$
\left\|\boldsymbol{u}-\boldsymbol{u}_{h}\right\|_{1}+\left\|\nabla^{h} p-\nabla^{h} p_{h}\right\|+\left\|\nabla^{h} p-\tilde{\boldsymbol{u}}_{h}\right\| \leq C h^{r},
$$

where $r=\min \left\{k, k_{\mathrm{v}}, k_{\mathrm{q}}+1\right\}$ and $\boldsymbol{u}$ and $p$ are the solution of the continuous problem (13) (without the nonlinear term).

Proof. The proof is essentially the same as in Theorem 3 in [10]. The main difference is due to the fact that the parameters $\alpha_{K}$ are now allowed to change from element to element. This only affects the following bound:

$$
\begin{aligned}
\| P_{h, 1}\left(\nabla^{h} q_{h}\right) & -P_{h, 1}\left(\nabla^{h} p_{h}\right) \|^{2} \\
& =\left(\nabla^{h} p-\nabla^{h} p_{h}, P_{h, 1}\left(\nabla^{h} q_{h}\right)-P_{h, 1}\left(\nabla^{h} p_{h}\right)\right) \\
& +\left(\nabla^{h} q_{h}-\nabla^{h} p, P_{h, 1}\left(\nabla^{h} q_{h}\right)-P_{h, 1}\left(\nabla^{h} p_{h}\right)\right) \\
& =: T_{1}+T_{2},
\end{aligned}
$$

for all functions $q_{h} \in Q_{h}$. The first term $T_{1}$ can be written as

$$
\begin{aligned}
T_{1}= & \sum_{K} \alpha_{K}\left(\nabla p-\nabla p_{h}, P_{h, 1}\left(\nabla^{h} q_{h}\right)-P_{h, 1}\left(\nabla^{h} p_{h}\right)\right) \\
= & \sum_{K}\left(\nabla p-\nabla p_{h}, \Pi\left(\bar{\alpha} P_{h, 1}\left(\nabla^{h} q_{h}\right)\right)-\Pi\left(\bar{\alpha} P_{h, 1}\left(\nabla^{h} p_{h}\right)\right)\right)_{K} \\
+ & \sum_{K}\left(\nabla p-\nabla p_{h}, \alpha_{K} P_{h, 1}\left(\nabla^{h} q_{h}\right)-\alpha_{K} P_{h, 1}\left(\nabla^{h} p_{h}\right)\right. \\
& \left.\quad-\Pi\left(\bar{\alpha} P_{h, 1}\left(\nabla^{h} q_{h}\right)\right)+\Pi\left(\bar{\alpha} P_{h, 1}\left(\nabla^{h} p_{h}\right)\right)\right)_{K} \\
(60)= & : T_{11}+T_{12} .
\end{aligned}
$$

From (21) (without the nonlinear term) we have that

$$
\begin{aligned}
T_{11} & =-a\left(\boldsymbol{u}-\boldsymbol{u}_{h}, \Pi\left(\bar{\alpha} P_{h, 1}\left(\nabla^{h} q_{h}\right)\right)-\Pi\left(\bar{\alpha} P_{h, 1}\left(\nabla^{h} p_{h}\right)\right)\right) \\
& \leq N_{a}\left\|\boldsymbol{u}-\boldsymbol{u}_{h}\right\|_{1}\left\|\Pi\left(\bar{\alpha} P_{h, 1}\left(\nabla^{h} q_{h}\right)\right)-\Pi\left(\bar{\alpha} P_{h, 1}\left(\nabla^{h} p_{h}\right)\right)\right\|_{1} .
\end{aligned}
$$


Using the same steps as those to arrive to (53) we get

$$
\begin{aligned}
& \left\|\Pi\left(\bar{\alpha} P_{h, 1}\left(\nabla^{h} q_{h}\right)\right)-\Pi\left(\bar{\alpha} P_{h, 1}\left(\nabla^{h} p_{h}\right)\right)\right\|_{1} \\
& \quad \leq C\left\|P_{h, 1}\left(\nabla^{h} q_{h}\right)-P_{h, 1}\left(\nabla^{h} p_{h}\right)\right\|,
\end{aligned}
$$

and therefore

$$
T_{11} \leq C\left\|\boldsymbol{u}-\boldsymbol{u}_{h}\right\|_{1}\left\|P_{h, 1}\left(\nabla^{h} q_{h}\right)-P_{h, 1}\left(\nabla^{h} p_{h}\right)\right\| .
$$

For $T_{12}$ in (60) we have that

$$
\begin{gathered}
T_{12}=\sum_{K}\left(\nabla^{h} p-\nabla^{h} p_{h}, P_{h, 1}\left(\nabla^{h} q_{h}\right)-\frac{1}{\alpha_{K}} \Pi\left(\bar{\alpha} P_{h, 1}\left(\nabla^{h} q_{h}\right)\right)\right. \\
\left.-P_{h, 1}\left(\nabla^{h} p_{h}\right)+\frac{1}{\alpha_{K}} \Pi\left(\bar{\alpha} P_{h, 1}\left(\nabla^{h} p_{h}\right)\right)\right)_{K},
\end{gathered}
$$

and using the same steps as in (56) we obtain

$$
T_{12} \leq \delta(h)\left\|\nabla^{h} p-\nabla^{h} p_{h}\right\|\left\|P_{h, 1}\left(\nabla^{h} q_{h}\right)-P_{h, 1}\left(\nabla^{h} p_{h}\right)\right\| .
$$

Using (60)-(62) in (59) we get

$$
\begin{aligned}
& \left\|P_{h, 1}\left(\nabla^{h} q_{h}\right)-P_{h, 1}\left(\nabla^{h} p_{h}\right)\right\| \\
& \quad \leq C\left\|\boldsymbol{u}-\boldsymbol{u}_{h}\right\|_{1}+\delta(h)\left\|\nabla^{h} p-\nabla^{h} p_{h}\right\|+\left\|\nabla^{h} p-\nabla^{h} q_{h}\right\| .
\end{aligned}
$$

The proof concludes as in Theorem 3 of [10], assuming $h$ to be sufficiently small (note that there the projection of the pressure gradient does not include the parameters $\alpha_{K}$ ).

We use now a duality argument to obtain pressure stability in $L^{2}(\Omega)$ and improved error estimates for the velocity and pressure, also in the space $L^{2}(\Omega)$, in a similar way to [4] for the GLS method. The shift used in these duality arguments needed for the velocity error estimates (not for the pressure) requires of more regularity of the problem than was needed up to now.

Theorem 3 Under the same assumptions as in Theorem 1, the approximate pressure $p_{h}$ satisfies:

$$
\left\|p_{h}\right\| \leq C \lambda\|\boldsymbol{f}\|_{-1} .
$$

If the solution $[\boldsymbol{u}, p]$ of the continuous problem is $k$-regular, with $k \geq 1$, then

$$
\left\|p-p_{h}\right\| \leq C h^{r}
$$


where $r=\min \left\{k, k_{\mathrm{v}}, k_{\mathrm{q}}+1\right\}$. Moreover, if the Stokes problem is regular then the approximate velocity $\boldsymbol{u}_{h}$ satisfies

$$
\left\|\boldsymbol{u}-\boldsymbol{u}_{h}\right\| \leq C h^{r+1} .
$$

Proof. We begin by the stability and error estimates for the pressure. Let $\gamma=0$ or 1 and consider $\boldsymbol{z} \in \boldsymbol{H}_{0}^{1}(\Omega)$ and $\eta \in L^{2}(\Omega)$ as the solution of the Stokes problem:

$$
\begin{aligned}
-\Delta \boldsymbol{z}+\nabla \eta & =0 \text { in } \Omega, \\
\nabla \cdot \boldsymbol{z} & =\left(\gamma p-p_{h}\right) \text { in } \Omega, \\
\boldsymbol{z} & =\mathbf{0} \text { on } \Gamma .
\end{aligned}
$$

Standard results for this problem yield (see [16]):

$$
\|\boldsymbol{z}\|_{1} \leq C\left\|\gamma p-p_{h}\right\|, \quad\|\eta\| \leq C\left\|\gamma p-p_{h}\right\| .
$$

If $\boldsymbol{z}_{h} \in V_{h, 0}$ is an approximation to $\boldsymbol{z}$ satisfying:

$$
\left\|\boldsymbol{z}-\boldsymbol{z}_{h}\right\|_{m, K} \leq C h_{K}^{1-m}\|\boldsymbol{z}\|_{1, K}, \quad K \in \mathcal{T}_{h},
$$

for $m=0,1$, we have:

$$
\begin{aligned}
\| \gamma p & -p_{h} \|^{2}=\left(\gamma p-p_{h}, \gamma p-p_{h}\right) \\
= & \left(\nabla \cdot \boldsymbol{z}, \gamma p-p_{h}\right) \\
= & \left(\nabla \cdot\left(\boldsymbol{z}-\boldsymbol{z}_{h}\right), \gamma p-p_{h}\right)-\left(\boldsymbol{z}_{h}, \nabla\left(\gamma p-p_{h}\right)\right) \\
= & -\left(\boldsymbol{z}-\boldsymbol{z}_{h}, \nabla\left(\gamma p-p_{h}\right)\right)+a\left(\gamma \boldsymbol{u}-\boldsymbol{u}_{h}, \boldsymbol{z}_{h}\right)+\gamma \lambda\left\langle\boldsymbol{f}, \boldsymbol{z}_{h}\right\rangle \\
= & \left.-\sum_{K} \frac{1}{\alpha_{K}}\left(\boldsymbol{z}-\boldsymbol{z}_{h}, \gamma \nabla^{h} p-\nabla^{h} p_{h}\right)\right)_{K} \\
& +a\left(\gamma \boldsymbol{u}-\boldsymbol{u}_{h}, \boldsymbol{z}_{h}-\boldsymbol{z}\right)+a\left(\gamma \boldsymbol{u}-\boldsymbol{u}_{h}, \boldsymbol{z}\right) \\
& +\gamma \lambda\left\langle\boldsymbol{f}, \boldsymbol{z}_{h}-\boldsymbol{z}\right\rangle+\gamma \lambda\langle\boldsymbol{f}, \boldsymbol{z}\rangle \\
\leq & \sum_{K} \frac{1}{\alpha_{K}}\left\|\boldsymbol{z}-\boldsymbol{z}_{h}\right\|_{0, K}\left\|\gamma \nabla^{h} p-\nabla^{h} p_{h}\right\|_{0, K} \\
& +C\left(\left\|\gamma \boldsymbol{u}-\boldsymbol{u}_{h}\right\|_{1}+\gamma \lambda\|\boldsymbol{f}\|_{-1}\right)\left(\left\|\boldsymbol{z}-\boldsymbol{z}_{h}\right\|_{1}+\|\boldsymbol{z}\|_{1}\right) \\
\leq & C \sum_{K}\|\boldsymbol{z}\|_{1, K}\left\|\gamma \nabla^{h} p-\nabla^{h} p_{h}\right\|_{0, K} \\
& +C\|\boldsymbol{z}\|_{1}\left(\left\|\gamma \boldsymbol{u}-\boldsymbol{u}_{h}\right\|_{1}+\gamma \lambda\|\boldsymbol{f}\|_{-1}\right) \\
(71) \leq & C\left(\left\|\gamma \nabla^{h} p-\nabla^{h} p_{h}\right\|+\left\|\gamma \boldsymbol{u}-\boldsymbol{u}_{h}\right\|_{1}+\gamma \lambda\|\boldsymbol{f}\|_{-1}\right)\left\|\gamma p-p_{h}\right\| .
\end{aligned}
$$

The stability estimate (63) for the pressure follows for $\gamma=0$ and Theorem 1 , whereas the error estimate (64) is obtained for $\gamma=1$ using (58). 
Let us prove now the estimate for the velocity. Let $\boldsymbol{y} \in \boldsymbol{H}^{2}(\Omega) \cap \boldsymbol{H}_{0}^{1}(\Omega)$ and $\xi \in H^{1}(\Omega) \cap Q$ be the solution of the regular problem:

$$
\begin{aligned}
-\Delta \boldsymbol{y}+\nabla \xi & =\boldsymbol{u}-\boldsymbol{u}_{h} \quad \text { in } \Omega, \\
\nabla \cdot \boldsymbol{y} & =0 \quad \text { in } \Omega, \\
\boldsymbol{y} & =\mathbf{0} \quad \text { on } \quad \Gamma,
\end{aligned}
$$

which satisfies

$$
\|\boldsymbol{y}\|_{2} \leq C\left\|\boldsymbol{u}-\boldsymbol{u}_{h}\right\|, \quad\|\xi\|_{1} \leq C\left\|\boldsymbol{u}-\boldsymbol{u}_{h}\right\| .
$$

Let $\boldsymbol{y}_{h} \in V_{h, 0}$ and $\xi_{h} \in Q_{h}$ be optimal order approximations to $\boldsymbol{y}$ and $\xi$, respectively, satisfying:

$$
\begin{aligned}
\left\|\boldsymbol{y}-\boldsymbol{y}_{h}\right\|_{m, K} & \leq C h_{K}^{2-m}\|\boldsymbol{y}\|_{2, K}, \\
\left\|\xi-\xi_{h}\right\|_{m, K} & \leq C h_{K}^{1-m}\|\xi\|_{1, K} .
\end{aligned}
$$

for $m=0,1$. We then have:

$$
\begin{aligned}
\left\|\boldsymbol{u}-\boldsymbol{u}_{h}\right\|^{2} & =\left(\boldsymbol{u}-\boldsymbol{u}_{h}, \boldsymbol{u}-\boldsymbol{u}_{h}\right) \\
& =a\left(\boldsymbol{y}, \boldsymbol{u}-\boldsymbol{u}_{h}\right)-b\left(\xi, \boldsymbol{u}-\boldsymbol{u}_{h}\right) \\
& =\left[a\left(\boldsymbol{y}-\boldsymbol{y}_{h}, \boldsymbol{u}-\boldsymbol{u}_{h}\right)-b\left(\xi-\xi_{h}, \boldsymbol{u}-\boldsymbol{u}_{h}\right)\right] \\
& +a\left(\boldsymbol{y}_{h}, \boldsymbol{u}-\boldsymbol{u}_{h}\right)-b\left(\xi_{h}, \boldsymbol{u}-\boldsymbol{u}_{h}\right)=: T_{1}+T_{2}+T_{3} .
\end{aligned}
$$

We bound each term separately:

$$
\begin{aligned}
T_{1} & =a\left(\boldsymbol{y}-\boldsymbol{y}_{h}, \boldsymbol{u}-\boldsymbol{u}_{h}\right)-b\left(\xi-\xi_{h}, \boldsymbol{u}-\boldsymbol{u}_{h}\right) \\
& \leq N_{a}\left\|\boldsymbol{y}-\boldsymbol{y}_{h}\right\|_{1}\left\|\boldsymbol{u}-\boldsymbol{u}_{h}\right\|_{1}+C\left\|\xi-\xi_{h}\right\|\left\|\boldsymbol{u}-\boldsymbol{u}_{h}\right\|_{1} \\
& \leq C\left\|\boldsymbol{u}-\boldsymbol{u}_{h}\right\|_{1}\left(h\|\boldsymbol{y}\|_{2}+h\|\xi\|_{1}\right) \\
& \leq C h\left\|\boldsymbol{u}-\boldsymbol{u}_{h}\right\|_{1}\left\|\boldsymbol{u}-\boldsymbol{u}_{h}\right\|
\end{aligned}
$$

by (76), (77) and the shift (75). From the original momentum equation (with $c=0$ ) we have:

$$
\begin{aligned}
T_{2} & =a\left(\boldsymbol{y}_{h}, \boldsymbol{u}-\boldsymbol{u}_{h}\right) \\
& =-\left(\nabla p-\nabla p_{h}, \boldsymbol{y}_{h}\right) \\
& =\sum_{K} \frac{1}{\alpha_{K}}\left(\nabla^{h} p-\nabla^{h} p_{h}, \boldsymbol{y}-\boldsymbol{y}_{h}\right)_{K} \\
& \leq C \sum_{K} \frac{1}{h_{K}}\left\|\nabla^{h} p-\nabla^{h} p_{h}\right\|_{0, K}\left\|\boldsymbol{y}-\boldsymbol{y}_{h}\right\|_{0, K} \\
& \leq C \sum_{K} h_{K}\|\boldsymbol{y}\|_{2, K}\left\|\nabla^{h} p-\nabla^{h} p_{h}\right\|_{0, K} \\
& \leq C h\left\|\boldsymbol{u}-\boldsymbol{u}_{h}\right\|\left\|\nabla^{h} p-\nabla^{h} p_{h}\right\|,
\end{aligned}
$$


by (76) and (75). Finally:

$$
\begin{aligned}
T_{3}= & -b\left(\xi_{h}, \boldsymbol{u}-\boldsymbol{u}_{h}\right) \\
= & \left(\nabla^{h} p-\nabla^{h} p_{h}, \nabla^{h} \xi_{h}\right)-\left(\nabla^{h} p-\tilde{\boldsymbol{u}}_{h}, \nabla^{h} \xi_{h}\right) \\
= & \left(\nabla^{h} p-\nabla^{h} p_{h}, \nabla^{h} \xi_{h}-\nabla^{h} \xi\right)+\left(\nabla^{h} p-\nabla^{h} p_{h}, \nabla^{h} \xi\right) \\
- & \left(\nabla^{h} p-\tilde{\boldsymbol{u}}_{h}, \nabla^{h} \xi_{h}-\nabla^{h} \xi\right)-\left(\nabla^{h} p-\tilde{\boldsymbol{u}}_{h}, \nabla^{h} \xi\right) \\
\leq & \sum_{K}\left(\left\|\nabla^{h} p-\nabla^{h} p_{h}\right\|_{0, K}+\left\|\nabla^{h} p-\tilde{\boldsymbol{u}}_{h}\right\|_{0, K}\right) \\
& \times\left(\left\|\nabla^{h} \xi_{h}-\nabla^{h} \xi\right\|_{0, K}+\left\|\nabla^{h} \xi\right\|_{0, K}\right) \\
\leq & C \sum_{K}\left(\left\|\nabla^{h} p-\nabla^{h} p_{h}\right\|_{0, K}+\left\|\nabla^{h} p-\tilde{\boldsymbol{u}}_{h}\right\|_{0, K}\right) h_{K}\|\xi\|_{1, K} \\
\leq & C\left(\left\|\nabla^{h} p-\nabla^{h} p_{h}\right\|+\left\|\nabla^{h} p-\tilde{\boldsymbol{u}}_{h}\right\|\right) h\left\|\boldsymbol{u}-\boldsymbol{u}_{h}\right\|,
\end{aligned}
$$

by (77) and (75). We obtain the error estimate for the velocity combining the above inequalities for $T_{1}, T_{2}$ and $T_{3}$.

\section{Stability and convergence II: Navier-Stokes equations}

In this section we extend the results of the previous section to the nonlinear Navier-Stokes equations using the theory of approximation of branches of nonsingular solutions of $[7,16]$. However, our first result concerns the case in which the uniqueness condition (14) holds. We show that this same condition ensures stability and uniqueness of solution of the discrete problem. To prove this we shall use as auxiliary problem a linearized form of it, namely, Picard's linearization. Denoting by a superscript the iteration counter, this problem is: given $\boldsymbol{u}_{h}^{0}$ arbitrary, for $i=1,2, \ldots$, find $\left[\boldsymbol{u}^{i}, p_{h}^{i}, \tilde{\boldsymbol{u}}_{h}^{i}\right] \in \boldsymbol{V}_{h, 0} \times Q_{h} \times \boldsymbol{V}_{h}$ such that

$$
\mathcal{B}_{(i)}\left(\lambda ; \boldsymbol{u}_{h}^{i}, p_{h}^{i}, \tilde{\boldsymbol{u}}_{h}^{i} ; \boldsymbol{v}_{h}, q_{h}, \tilde{\boldsymbol{v}}_{h}\right)=\lambda\left\langle\boldsymbol{f}, \boldsymbol{v}_{h}\right\rangle
$$

for all $\left[\boldsymbol{v}_{h}, q_{h}, \tilde{\boldsymbol{v}}_{h}\right] \in \boldsymbol{V}_{h, 0} \times Q_{h} \times \boldsymbol{V}_{h}$. Here, $\mathcal{B}_{(i)}$ is the bilinear form obtained from $\mathcal{B}$ using $c\left(\boldsymbol{u}_{h}^{i-1}, \boldsymbol{u}_{h}^{i}, \boldsymbol{v}_{h}\right)$ as linearization of the nonlinear term.

As a by-product of the following theorem we shall have convergence of Picard's iterates, a property that does not hold when the solution to the nonlinear problem (13) is not unique (an alternative proof of the following result is to proceed the other way around, showing that when (14) holds the nonlinear operator associated to the variational problem (21)-(23) is contractive, and thus Banach's fixed point theorem implies that there is a unique solution to which Picard's linearization scheme converges). 
Theorem 4 Suppose that the family $\left\{\mathcal{T}_{h}\right\}_{h>0}$ of finite element partitions is non-degenerate, continuously graded and such that (45) and (46) hold. Suppose also that the uniqueness condition (14) holds. Then, for $h$ small enough, there exists a unique solution to problem (26) that verifies the stability estimate

$$
\left\|\boldsymbol{u}_{h}\right\|_{1}+\left\|\nabla^{h} p_{h}\right\|+\left\|\tilde{\boldsymbol{u}}_{h}\right\| \leq C \lambda\|\boldsymbol{f}\|_{-1},
$$

for a constant $C$ independent of $h$.

Proof. We split the proof in three steps:

Step 1: The solution of the linear problem (79) exists, is unique and each iterate satisfies the stability estimate (80).

Since problem (79) is linear and finite dimensional, it is enough to prove the stability estimate (80). Due to the skew-symmetry of $c$ (cf. (8)), this can be done exactly as for the Stokes problem, replacing $\mathcal{B}_{\text {lin }}$ by $\mathcal{B}_{(i)}$. The proof of Theorem 1 can be repeated here, the only difference being the bound for $T_{1}$ in (52). This term is now

$$
\begin{aligned}
T_{1} & =\lambda\left\langle\boldsymbol{f}, \Pi\left(\bar{\alpha} P_{h, 1}\left(\nabla^{h} p_{h}^{i}\right)\right)\right\rangle-a\left(\boldsymbol{u}_{h}^{i}, \Pi\left(\bar{\alpha} P_{h, 1}\left(\nabla^{h} p_{h}^{i}\right)\right)\right) \\
& -\lambda c\left(\boldsymbol{u}_{h}^{i-1}, \boldsymbol{u}_{h}^{i}, \Pi\left(\bar{\alpha} P_{h, 1}\left(\nabla^{h} p_{h}^{i}\right)\right)\right),
\end{aligned}
$$

and, using the uniqueness condition (14) and the bounds for the velocity iterates (i.e., bound (49) for $\boldsymbol{u}_{h}^{i}$ and $\boldsymbol{u}_{h}^{i-1}$ ), the last term can be bounded by

$$
\begin{aligned}
& \lambda c\left(\boldsymbol{u}_{h}^{i-1}, \boldsymbol{u}_{h}^{i}, \Pi\left(\bar{\alpha} P_{h, 1}\left(\nabla^{h} p_{h}^{i}\right)\right)\right) \\
& \quad \leq \lambda N_{c}\left\|\boldsymbol{u}_{h}^{i-1}\right\|_{1}\left\|\boldsymbol{u}_{h}^{i}\right\|_{1}\left\|\Pi\left(\bar{\alpha} P_{h, 1}\left(\nabla^{h} p_{h}^{i}\right)\right)\right\|_{1} \\
& \quad \leq \lambda\|\boldsymbol{f}\|_{-1}\left\|\Pi\left(\bar{\alpha} P_{h, 1}\left(\nabla^{h} p_{h}^{i}\right)\right)\right\| .
\end{aligned}
$$

and now we can proceed as in Theorem 1.

Step 2: Picard's iterates converge to a solution of the nonlinear problem (26).

Subtracting Eqs. (79) for $i$ and $i-1$ and taking as test functions $\boldsymbol{v}_{h}=$ $\boldsymbol{u}_{h}^{i}-\boldsymbol{u}_{h}^{i-1}, q_{h}=p_{h}^{i}-p_{h}^{i-1}$ and $\tilde{\boldsymbol{v}}_{h}=\tilde{\boldsymbol{u}}_{h}^{i}-\tilde{\boldsymbol{u}}_{h}^{i-1}$, we get

$$
\begin{aligned}
& a\left(\boldsymbol{u}_{h}^{i}-\boldsymbol{u}_{h}^{i-1}, \boldsymbol{u}_{h}^{i}-\boldsymbol{u}_{h}^{i-1}\right)+\left\|\nabla^{h}\left(p_{h}^{i}-p_{h}^{i-1}\right)-\left(\tilde{\boldsymbol{u}}_{h}^{i}-\tilde{\boldsymbol{u}}_{h}^{i-1}\right)\right\|^{2} \\
& \quad+\lambda c\left(\boldsymbol{u}_{h}^{i-1}-\boldsymbol{u}_{h}^{i-2}, \boldsymbol{u}_{h}^{i}, \boldsymbol{u}_{h}^{i}-\boldsymbol{u}_{h}^{i-1}\right)=0 .
\end{aligned}
$$

From the coercivity of $a$ and the bound for $\boldsymbol{u}_{h}^{i}$ we have that

$$
\begin{aligned}
K_{a}\left\|\boldsymbol{u}_{h}^{i}-\boldsymbol{u}_{h}^{i-1}\right\|_{1}^{2} & \leq \lambda N_{c}\left\|\boldsymbol{u}_{h}^{i-1}-\boldsymbol{u}_{h}^{i-2}\right\|_{1}\left\|\boldsymbol{u}_{h}^{i}\right\|_{1}\left\|\boldsymbol{u}_{h}^{i}-\boldsymbol{u}_{h}^{i-1}\right\|_{1} \\
& \leq \lambda^{2} N_{c} K_{a}^{-1}\|\boldsymbol{f}\|_{-1}\left\|\boldsymbol{u}_{h}^{i-1}-\boldsymbol{u}_{h}^{i-2}\right\|_{1}\left\|\boldsymbol{u}_{h}^{i}-\boldsymbol{u}_{h}^{i-1}\right\|_{1} .
\end{aligned}
$$


Using inductively this inequality and the definition of $\chi$ in (14) we have that

$$
\left\|\boldsymbol{u}_{h}^{i}-\boldsymbol{u}_{h}^{i-1}\right\|_{1} \leq C \chi^{i} .
$$

Since $\chi<1$ this proves convergence of the velocities. Using this in (81) we find that

$$
\begin{aligned}
\left\|P_{h, 3}\left(\nabla^{h}\left(p_{h}^{i}-p_{h}^{i-1}\right)\right)\right\|^{2} & =\left\|\nabla^{h}\left(p_{h}^{i}-p_{h}^{i-1}\right)-\left(\tilde{\boldsymbol{u}}_{h}^{i}-\tilde{\boldsymbol{u}}_{h}^{i-1}\right)\right\|^{2} \\
& \leq \lambda N_{c}\left\|\boldsymbol{u}_{h}^{i-1}-\boldsymbol{u}_{h}^{i-2}\right\|_{1}\left\|\boldsymbol{u}_{h}^{i}\right\|_{1}\left\|\boldsymbol{u}_{h}^{i}-\boldsymbol{u}_{h}^{i-1}\right\|_{1} \\
& \leq C \chi^{2 i},
\end{aligned}
$$

from where

$$
\left\|P_{h, 3}\left(\nabla^{h}\left(p_{h}^{i}-p_{h}^{i-1}\right)\right)\right\| \leq C \chi^{i} .
$$

To obtain a bound for $\left\|P_{h, 1}\left(\nabla^{h}\left(p_{h}^{i}-p_{h}^{i-1}\right)\right)\right\|$ we proceed as in Theorem 1 to obtain the bound for $\left\|P_{h, 1}\left(\nabla^{h} p_{h}\right)\right\|$. Equation (51) is also valid replacing $p_{h}$ by $p_{h}^{i}-p_{h}^{i-1}$. The new term $T_{1}$ is now

$$
\begin{aligned}
& T_{1}=-a\left(\boldsymbol{u}_{h}^{i}-\boldsymbol{u}_{h}^{i-1}, \Pi\left(\bar{\alpha} P_{h, 1}\left(\nabla^{h} p_{h}^{i}\right)\right)-\Pi\left(\bar{\alpha} P_{h, 1}\left(\nabla^{h} p_{h}^{i-1}\right)\right)\right) \\
& -\lambda c\left(\boldsymbol{u}_{h}^{i-1}-\boldsymbol{u}_{h}^{i-2}, \boldsymbol{u}^{i-1}, \Pi\left(\bar{\alpha} P_{h, 1}\left(\nabla^{h} p_{h}^{i}\right)\right)-\Pi\left(\bar{\alpha} P_{h, 1}\left(\nabla^{h} p_{h}^{i-1}\right)\right)\right) \\
& -\lambda c\left(\boldsymbol{u}^{i-1}, \boldsymbol{u}_{h}^{i}-\boldsymbol{u}_{h}^{i-1}, \Pi\left(\bar{\alpha} P_{h, 1}\left(\nabla^{h} p_{h}^{i}\right)\right)-\Pi\left(\bar{\alpha} P_{h, 1}\left(\nabla^{h} p_{h}^{i-1}\right)\right)\right) .
\end{aligned}
$$

Using the continuity of $a$ and $c$, the bound for $\boldsymbol{u}_{h}^{i}$ and (82), as well as (53) with $p_{h}$ replaced by $p_{h}^{i}-p_{h}^{i-1}$, we obtain that

$$
T_{1} \leq C \chi^{i}\left\|P_{h, 1}\left(\nabla^{h}\left(p_{h}^{i}-p_{h}^{i-1}\right)\right)\right\| .
$$

For the new term $T_{2}$ we have, using the same steps as in (56),

$$
\begin{aligned}
T_{2} & \leq C \delta(h)\left\|P_{h, 1}\left(\nabla^{h}\left(p_{h}^{i}-p_{h}^{i-1}\right)\right)\right\|^{2} \\
& +C\left\|P_{h, 1}\left(\nabla^{h}\left(p_{h}^{i}-p_{h}^{i-1}\right)\right)\right\|\left\|P_{h, 3}\left(\nabla^{h}\left(p_{h}^{i}-p_{h}^{i-1}\right)\right)\right\| .
\end{aligned}
$$

Using the bound (83) for $P_{h, 3}\left(\nabla^{h}\left(p_{h}^{i}-p_{h}^{i-1}\right)\right)$ and (84), for $h$ small enough we have that,

$$
\left\|P_{h, 1}\left(\nabla^{h}\left(p_{h}^{i}-p_{h}^{i-1}\right)\right)\right\| \leq C \chi^{i} .
$$

Convergence of the pressure follows now from (83), (85) and the weak inf-sup condition, and convergence of $\tilde{\boldsymbol{u}}^{i}$ follows from the fact that $\tilde{\boldsymbol{u}}_{h}^{i}=$ $P_{h, 12}\left(\nabla^{h} p_{h}^{i}\right)$. 
Step 3: Problem (26) admits a unique solution.

Let $\left(\boldsymbol{u}_{h, 1}, p_{h, 1}, \tilde{\boldsymbol{u}}_{h, 1}\right)$ and $\left(\boldsymbol{u}_{h, 2}, p_{h, 2}, \tilde{\boldsymbol{u}}_{h, 2}\right)$ be two solutions of problem (26) and $\left(\overline{\boldsymbol{u}}_{h}, \bar{p}_{h}, \overline{\tilde{\boldsymbol{u}}}_{h}\right)$ their difference. It is easy to see that $\boldsymbol{u}_{h, 1}$ and $\boldsymbol{u}_{h, 2}$ satisfy the bound (80) and that

$$
\begin{aligned}
\mathcal{B}\left(\overline{\boldsymbol{u}}_{h}, \bar{p}_{h}, \overline{\tilde{\boldsymbol{u}}}_{h} ; \overline{\boldsymbol{u}}_{h}, \bar{p}_{h}, \overline{\tilde{\boldsymbol{u}}}_{h}\right) & =a\left(\overline{\boldsymbol{u}}_{h}, \overline{\boldsymbol{u}}_{h}\right)+\left\|\nabla^{h} \bar{p}_{h}-\overline{\tilde{\boldsymbol{u}}}_{h}\right\| \\
& =-\lambda c\left(\overline{\boldsymbol{u}}_{h}, \boldsymbol{u}_{h, 1}, \overline{\boldsymbol{u}}_{h}\right) .
\end{aligned}
$$

The coercivity of $a$, the continuity of $c$ and the bound (80) for $\boldsymbol{u}_{h, 1}$ imply that

$$
\left\|\overline{\boldsymbol{u}}_{h}\right\|_{1}^{2} \leq \chi\left\|\overline{\boldsymbol{u}}_{h}\right\|_{1}^{2},
$$

and, since $\chi<1, \overline{\boldsymbol{u}}_{h}=\mathbf{0}$. Also, $\bar{p}_{h}=0$ and $\overline{\tilde{\boldsymbol{u}}}_{h}=\mathbf{0}$ follows from the fact that these variables are solution of a linear homogeneous problem and satisfy the stability estimates obtained in the linear case.

Let us consider know the general case in which the uniqueness condition (14) does not hold. Regardless of the behavior of the continuous problem, it can be shown using exactly the same arguments as in [7] (also used in [22]) that problem (21)-(23) has solution. However, we consider directly the situation in which these solutions exist and approximate those of the continuous problem. For that, we need to recast it in the following abstract form. Let $\Lambda$ be a compact subset of $\mathbb{R}^{+}$and for each $\lambda \in \Lambda$ consider the mappings

$$
\boldsymbol{V} \times Q \stackrel{G}{\longrightarrow} \boldsymbol{V}^{\prime} \stackrel{T}{\longrightarrow} \boldsymbol{V} \times Q,
$$

where

$$
G(\lambda ; \boldsymbol{v}, q):=\lambda\left[(\boldsymbol{v} \cdot \nabla) \boldsymbol{v}+\frac{1}{2}(\nabla \cdot \boldsymbol{v}) \boldsymbol{v}-\boldsymbol{f}\right],
$$

and $[\boldsymbol{u}, p]:=T \boldsymbol{g}$ is the solution of the continuous Stokes problem

$$
a(\boldsymbol{u}, p)-b(p, \boldsymbol{v})+b(q, \boldsymbol{u})=\langle\boldsymbol{g}, \boldsymbol{v}\rangle, \quad \forall[\boldsymbol{v}, q] \in \boldsymbol{V} \times Q .
$$

Clearly, the solution of problem (13) is $[\boldsymbol{u}, p]=-T G(\lambda ; \boldsymbol{u}, p)$, that is, the solution of

$$
F(\lambda ; \boldsymbol{u}, p):=[\boldsymbol{u}, p]+T G(\lambda ; \boldsymbol{u}, p)=0 .
$$

Likewise, the discrete Navier-Stokes problem can be written in a form similar to (86) with the help of the operators

$$
\boldsymbol{V}_{h} \times Q_{h} \stackrel{G_{h}}{\longrightarrow} V^{\prime} \stackrel{T_{h}}{\longrightarrow} V_{h} \times Q_{h},
$$


where $G_{h}\left(\lambda ; \boldsymbol{v}_{h}, q_{h}\right):=G\left(\lambda ; \boldsymbol{v}_{h}, q_{h}\right)$ and $\left[\boldsymbol{u}_{h}, p_{h}\right]:=T_{h} \boldsymbol{g}$ is the solution of the discrete Stokes problem 'condensing' the pressure gradient projection:

$$
a\left(\boldsymbol{u}_{h}, p_{h}\right)-b\left(p_{h}, \boldsymbol{v}_{h}\right)+b\left(q_{h}, \boldsymbol{u}_{h}\right)+\left(P_{h, 3}\left(\nabla^{h} p_{h}\right), \nabla^{h} q_{h}\right)=\left\langle\boldsymbol{g}, \boldsymbol{v}_{h}\right\rangle,
$$

for all $\left[\boldsymbol{v}_{h}, q_{h}\right] \in \boldsymbol{V}_{h} \times Q_{h}$. The solution $\left[\boldsymbol{u}_{h}, p_{h}\right]$ of problem (21)-(23) is the same as the solution of

$$
F_{h}\left(\lambda ; \boldsymbol{u}_{h}, p_{h}\right):=\left[\boldsymbol{u}_{h}, p_{h}\right]+T_{h} G_{h}\left(\lambda ; \boldsymbol{u}_{h}, p_{h}\right)=0 .
$$

Let $D F(\lambda ; \boldsymbol{u}, p)$ denote the Fréchet derivative of $F$ with respect to $[\boldsymbol{u}, p]$. A curve $\{(\lambda,[\boldsymbol{u}(\lambda), p(\lambda)]) ; \lambda \in \Lambda\}$ is called a branch of nonsingular solutions of (86) if $[\boldsymbol{u}(\lambda), p(\lambda)]$ is solution of this problem for all $\lambda \in \Lambda$, the map $\lambda \mapsto[\boldsymbol{u}(\lambda), p(\lambda)]$ is continuous and $D F(\lambda ; \boldsymbol{u}(\lambda), p(\lambda))$ is a homeomorphism of $\boldsymbol{V} \times Q$.

The following results shows that if a branch of nonsingular solutions of (86) is regular enough, then problem (87) has also a unique branch of nonsingular solutions which gives an approximation of optimal order to it:

Theorem 5 Suppose that the family $\left\{\mathcal{T}_{h}\right\}_{h>0}$ of finite element partitions is non-degenerate, continuously graded and such that (45) and (46) hold. Assume also that $\{(\lambda,[\boldsymbol{u}(\lambda), p(\lambda)]) ; \lambda \in \Lambda\}$ is a branch of nonsingular solutions of $(86)$ such that $\lambda \mapsto[\boldsymbol{u}(\lambda), p(\lambda)] \in \boldsymbol{V} \cap \boldsymbol{H}^{k+1}(\Omega) \times Q \cap H^{k}(\Omega)$ is continuous for a certain integer $k \geq 1$. Then, for $h$ small enough, there exists a unique branch of nonsingular solutions $\left\{\left(\lambda,\left[\boldsymbol{u}_{h}(\lambda), p_{h}(\lambda)\right]\right) ; \lambda \in\right.$ 1\} of problem (87) which satisfies

$$
\left\|\boldsymbol{u}(\lambda)-\boldsymbol{u}_{h}(\lambda)\right\|_{1}+\left\|p(\lambda)-p_{h}(\lambda)\right\| \leq C(\lambda) h^{r},
$$

for all $\lambda \in \Lambda$, where $r=\min \left\{k, k_{\mathrm{v}}, k_{\mathrm{q}}+1\right\}$ and $C(\lambda)$ depends on $\lambda$.

Proof. As in Theorem IV.4.1 of [16], the proof simply consists in checking that the assumptions of the abstract approximation result IV.3.3 of this reference are satisfied. First, we know that $T$ is a bounded linear operator from $\boldsymbol{V}^{\prime}$ to $\boldsymbol{V} \times Q$. By virtue of Theorem 1 and the pressure stability estimate (63) in Theorem 3, $T_{h}$ is also a bounded linear operator from $\boldsymbol{V}^{\prime}$ to $\boldsymbol{V}_{h} \times Q_{h}$ (endowing this space with the same norm as $\boldsymbol{V} \times Q$ ). Thus,

$$
T \in \mathcal{L}\left(\boldsymbol{V}^{\prime}, \boldsymbol{V} \times Q\right), \quad T_{h} \in \mathcal{L}\left(\boldsymbol{V}^{\prime}, \boldsymbol{V}_{h} \times Q_{h}\right) .
$$

On the other hand, $G$ is a $\mathcal{C}^{\infty}$ map whose Fréchet derivative with respect to $[\boldsymbol{u}, p], D G(\lambda ; \boldsymbol{u}, p)$, maps $\boldsymbol{V} \times Q$ to $\boldsymbol{V}^{\prime}$ for each $[\boldsymbol{u}, p] \in \boldsymbol{V} \times Q$ and is given by

$$
\begin{aligned}
& D G(\lambda ; \boldsymbol{u}, p) \cdot[\boldsymbol{v}, q] \\
& \quad=\lambda\left[(\boldsymbol{v} \cdot \nabla) \boldsymbol{u}+(\boldsymbol{u} \cdot \nabla) \boldsymbol{v}+\frac{1}{2}(\nabla \cdot \boldsymbol{v}) \boldsymbol{u}+\frac{1}{2}(\nabla \cdot \boldsymbol{u}) \boldsymbol{v}\right] .
\end{aligned}
$$


Furthermore, Sobolev's embedding theorem and Hölder's inequality imply that in fact $D G(\lambda ; \boldsymbol{u}, p) \cdot[\boldsymbol{v}, q] \in Z:=L^{3 / 2}(\Omega)$ whenever $\boldsymbol{u}, \boldsymbol{v} \in \boldsymbol{V}$. Therefore, for $G$ we have

$$
G \in \mathcal{C}^{\infty}\left(\boldsymbol{V} \times Q, \boldsymbol{V}^{\prime}\right), \quad D G(\lambda ; \boldsymbol{u}, p) \in \mathcal{L}(\boldsymbol{V} \times Q, Z) .
$$

On the other hand, Theorem 2 and (64) in Theorem 3 imply

$$
\left\|\left(T-T_{h}\right) \boldsymbol{g}\right\|_{\boldsymbol{V} \times Q} \leq C h^{r} \quad \forall \boldsymbol{g} \in \boldsymbol{V}^{\prime} .
$$

Since $Z=L^{3 / 2}(\Omega)$ is compactly embedded in $\boldsymbol{V}^{\prime}=\boldsymbol{H}^{-1}(\Omega)$, this last estimate implies

$$
\lim _{h \rightarrow 0}\left\|T-T_{h}\right\|_{\mathcal{L}(Z, \boldsymbol{V} \times Q)}=0 .
$$

Properties (89)-(92) are precisely the assumptions needed to apply Theorem IV.3.3 in [16], from where estimate (88) follows. The dependence of $C(\lambda)$ with $\lambda$ appears through the inverse of the homeomorphism $D F(\lambda ; \boldsymbol{u}(\lambda), p(\lambda))$.

Finally, optimal $L^{2}$ estimates for the velocity can be obtained if the Stokes problem is regular. The following result can be proved adapting the proof of Theorem IV.4.2 in [16] as done above in Theorem 5:

Theorem 6 Under the same assumptions as in Theorem 5, if, in addition, the Stokes problem is regular, then the error estimate

$$
\left\|\boldsymbol{u}-\boldsymbol{u}_{h}\right\| \leq C(\lambda) h^{r+1}
$$

holds for all $\lambda \in \Lambda$.

\section{References}

1. P.B. Bochev, Z. Cai, T.A. Manteuffel and S.F. McCormick. Analysis of velocity-flux first-order system least-squares principles for the Navier-Stokes equations: Part I. SIAM J. Numerical Analy. 35, 990-1009 (1998)

2. D. Boffi. Three-dimensional finite element method for the Stokes problem SIAM J. Numerical Analy. 34, 664-670 (1997)

3. S.C. Brenner and L.R. Scott. The mathematical theory of finite element methods. Springer, Berlin Heidelberg New York, 1994

4. F. Brezzi and J. Douglas. Stabilized mixed methods for the Stokes problem. Numerische Mathematik 53, 225-235 (1988)

5. F. Brezzi and R. Falk. Stability of higher-order Taylor-Hood elements. SIAM J. Numerical Analy. 28, 581-590 (1991)

6. F. Brezzi and M. Fortin. Mixed and hybrid finite element methods. Springer, Berlin Heidelberg New York, 1991 
7. F. Brezzi, J. Rappaz, and P.A. Raviart. Finite dimensional approximation of nonlinear problems. Part I: Branches of non-singular solutions. Numerische Mathematik 36, $1-25(1981)$

8. G.F. Carey and R. Krishnan. Penalty finite element method for the Navier-Stokes equations. Comput. Methods Appl. Mech. Eng. 42, 183-224 (1984)

9. T. Chacón A term by term stabilization algorithm for the finite element solution of incompressible flow problems. Numerische Mathematik 79, 283-319 (1998)

10. R. Codina and J. Blasco. A finite element formulation for the Stokes problem allowing equal velocity-pressure interpolation. Comput. Methods Appl. Mech. Eng. 143, 373391 (1997)

11. R. Codina and J. Blasco. Stabilized finite element method for the transient NavierStokes equations based on a pressure gradient projection Comput. Methods Appl. Mech. Eng. 182, 277-300 (2000)

12. J. Douglas and J. Wang. An absolutely stabilized finite element method for the Stokes problem. Math. Comput. 52, 495-508 (1989)

13. L.P. Franca and S.L. Frey Stabilized finite element methods: II. The incompressible Navier-Stokes equations. Comput. Methods Appl. Mech. Eng. 99, 209-233 (1992)

14. L. Franca, T.J.R. Hughes, A.F.D. Loula, and I. Miranda. A new family of stable elements for nearly incompressible elasticity based on a mixed Petrov-Galerkin finite element formulation. Numerische Mathematik 53, 123-141 (1988)

15. L. Franca and R. Stenberg. Error analysis of some Galerkin least-squares methods for the elasticity equations. SIAM J. Numerical Analy. 28, 1680-1697 (1991)

16. V. Girault and P.A. Raviart. Finite element methods for Navier-Stokes equations. Springer, Berlin Heidelberg, New York, 1986

17. T.J.R. Hughes, L.P. Franca, and M. Balestra. A new finite element formulation for computational fluid dynamics: V. Circumventing the Babuška-Brezzi condition: a stable Petrov-Galerkin formulation for the Stokes problem accommodating equal-order interpolations. Comput. Methods Appl. Mech. Eng. 59, 85-99 (1986)

18. R. Stenberg. Error analysis of some finite element methods for the Stokes problem. Math. Comput. 54, 495-508 (1990)

19. R. Stenberg. A technique for analysing finite element methods for viscous incompressible flow. Int. J. Num. Methods Fluids 11, 935-948 (1990)

20. R. Temam. Navier-Stokes equations. North-Holland, 1984

21. L. Tobiska and G. Lube. A modified streamline-diffusion method for solving the stationary Navier-Stokes equations. Numerische Mathematik 59, 13-29 (1991)

22. L. Tobiska and R. Verfürth. Analysis of a streamline diffusion finite element method for the Stokes and Navier-Stokes equations. SIAM J. Numerical Analy. 33, 107-127 (1996) 